\title{
Original
}

\section{Prostatectomia radical robótica: Análisis después de 100 casos en la Fundació Puigvert}

\author{
Humberto Villavicencio Mavrich, Salvador Esquena Fernández, José Salvador Bayarri, \\ N. De Graeve, Juan J. Gómez Ruiz, P. Escovar Lariva, Juan Palou Redorta
}

Servicio de Urología. Fundació Puigvert. Barcelona.

\begin{abstract}
Resumen
Objetivo: Realizar un análisis de los primeros 100 casos de nuestra serie de prostatectomía radical robótica con Da Vinci (PRRdaV) realizadas en nuestro Centro, que corresponden a la primera serie en España.

Material y Métodos: Se realiza un estudio retrospectivo de las 100 primeras PRRdaV consecutivas realizadas en la Fundació Puigvert entre Julio de 2005 y Enero de 2007. El procedimiento fue realizado por 5 cirujanos distintos, siendo la curva de aprendizaje para todos ellos. El abordaje fue transperitoneal en todos los casos. Se analizan, tiempo operatorio, pérdidas hemáticas, tasa de conversión, complicaciones intra y postoperatorias, estancia hospitalaria y días de sonda vesical. También se revisan las tasas y la localización de los márgenes positivos, así como los resultados funcionales, con un seguimiento medio de 10,3 meses.

Resultados: La media de tiempo operatorio fue de 180 minutos (100-310) y la pérdida hemática media de $210 \mathrm{~mL}$ (100390). En 2 casos se realizó transfusión postoperatoria. No se presentaron complicaciones intraoperatorias, y tampoco ninguna reconversión. Como acontecimientos postoperatorios destacan un síndrome compartimental, una retención aguda urinaria tras retirada de sonda vesical, y unas parestesias por compresión del plexo braquial. La estancia hospitalaria media fue 3,7 días (2-21). Se obtuvieron 21 casos con márgenes quirúrgicos positivos (21\%). La localización más frecuente fue posterolateral. El 69\% fueron continentes de forma precoz ( $<3$ meses), el $91 \%$ lo fueron a los 9 meses, el $9 \%$ restante requiere de compresa de seguridad. Referente a la función sexual, el 62\% conservan potencia y el 38\% presentaron disfunción eréctil postoperatoria.

Conclusiones: La PRRdaV es un procedimiento seguro, reproducible y que ofrece unos resultados oncológicos y funcionales muy prometedores con un abordaje mínimamente invasivo. A pesar de incluir la curva de aprendizaje de 5 cirujanos, obtenemos una excelente tasa de continencia, y una buena función sexual. La mejora individual, con mayor experiencia, así como un mayor seguimiento, permitirá valorar la evolución de la técnica, así como de sus resultados.
\end{abstract}

Palabras clave: Prostatectomía radical robótica. Da Vinci. Cáncer de próstata.

\section{Robotic radical prostatectomy: first 100 patients in Fundació Puigvert}

\section{Abstract}

Objective: We present the 100 first robotic radical prostatectomy with Da Vinci (RRPdaV), corresponding to the first experience in Spain.

Methods: We reviewed the first 100 patients that underwent transperitoneal RRPda performed in Fundació Puigvert between July 2005 and January 2007. All cases were performed by 5 surgeons, being the learning curve for all of them. We analyzed surgical time, blood loss, conversion rate, intra and postoperative complications, hospital stay and days of bladder catheterization. Also, rates and location of surgical margins, as well as functional outcomes with an average follow up of 10.3 months.

Results: Mean operating time was 180 minutes (100-310) and blood loss $210 \mathrm{~mL}$ (100-390). Blood transfusion was required in 2 cases. There were no intraoperative complications and neither any conversion to open surgery. There were 3 outstanding postoperative events, a compartmentalize syndrome, an acute urinary retention after removal of urethral catheter, and a paresthesias due to brachial plexus compression. Mean hospital stay were 3.7 days. (2-21). We had 21 cases of positive surgical margins (21\%). The most frequent location was posterior lateral. 69 of 100 patients $(69 \%)$ reached early $(<3$ months) total continence, $91 \%$ achieved in 9 months, and remaining 9\% required use of at least one pad. Concerning to sexual function, 13 of 100 patients (13\%) had preoperative erectile dysfunction, of remaining cases, $62 \%$ preserved potency at review, and $38 \%$ had postoperative erectile dysfunction.

Conclusions: RRPDAv is a safe and reproducible procedure, and offers promising oncological and functional results with a minimal invasive technique. In spite of include the learning curve of 5 surgeons; we obtain an excellent rate of continence, and an acceptable sexual function. The individual improvement, with more experience, and a longer follow-up, will allow to value evolution of the technique, and it results.

Keywords: Robotic radical prostatectomy. Da Vinci. Prostate cancer. 
$\mathrm{E}^{1}$ cáncer de próstata $(\mathrm{CaP})$ es la segunda causa principal de muerte relacionada con el cáncer en los varones de países occidentales. Los datos de incidencia anual oscilaron en el año 2000 entre 19 y 55/100.000 varones ${ }^{1}$. En los próximos años se convertirá en el cáncer más frecuente en hombres ${ }^{2}$. Según la base de datos de National Cancer Institute Surveillance, Epidemiology and End Results Program (SEER), los hombres de edades comprendidas entre 65 y 74 años son los que tienen mayor riesgo de ser diagnosticados de CaP, mientras que la tasa de mortalidad más elevada corresponde a los hombres de 75-84 años (www.seer.cancer.gov). Destaca que, actualmente el 91\% de los casos de CaP se diagnostican en estadio localizado. Existe una supervivencia absoluta específica del 100\% del CaP a 5 años cuando los pacientes presentan una neoplasia localizada frente a sólo un 33,3\% cuando hay metástasis ${ }^{1}$.

La prostatectomía radical (PR) es el tratamiento estándar para el cáncer de próstata localizado en pacientes jóvenes ${ }^{3}$. Después de los abordajes abiertos y laparoscópicos, la prostatectomía radical robótica con Da Vinci (PRRdaV) ha sido el siguiente paso en el camino de la cirugía mínimamente invasiva del CaP. El sistema Da Vinci (Intuitive Surgical, Sunnyvale, CA, EE.UU) permite una excelente maniobrabilidad para una técnica mínimamente invasiva, una postura más cómoda y ergonómica para el cirujano, una ventajosa visión tridimensional, y todo esto combinado con una reducida curva de aprendizaje, comparado con la laparoscopia. Además, su facilidad de uso permite una meticulosa disección que permite mejorar los resultados oncológicos y funcionales $^{4,5}$.

Después que en el año 2000 la primera PRRdaV la realizara el Dr. J. Binder en Frankfurt ${ }^{6}$, en noviembre del mismo año, el Dr. M. Menon y su equipo del Vattikuti Institute de Urología de Detroit, describieron la técnica original transperitoneal ${ }^{7,8}$, y recientemente, ha publicado la mayor serie de la literatura con $2.766 \operatorname{casos}^{9}$. La primera PRRdaV en España la realizó el Dr. H. Villavicencio en Julio de 2005 en la Fundació Puigvert de Barcelona ${ }^{10}$, que posteriormente describió su curva de aprendizaje en $20 \operatorname{casos}^{11}$.

En este artículo presentamos los resultados de los primeros 100 casos de PRRdaV en la Fundació Puigvert, siendo la primera serie y la mayor realizada en España.

\section{MATERIAL Y MÉTODOS}

Entre julio de 2005 y enero de 2007, se realizaron en nuestra Institución las primeras 100 PRRdaV en pacientes con cáncer de próstata localizado. Los procedimientos fueron ejecutados por 5 cirujanos, siendo la curva de aprendizaje de PRRdaV para todos ellos, siendo 4 los que disponían de experiencia laparoscópica previa. Aunque en la actualidad nuestra institución cuenta con más de 250 procedimientos, se revisan los 100 primeros, después de la experiencia acumulada hasta hoy.

En todos los casos se ha utilizado la técnica VIP (Vattikuti Institute Prostatectomy) ${ }^{12}$, usando los 4 brazos del robot. Se ha realizado un acceso transperitoneal, posicionando al paciente en posición de litotomía con Trendelenburg. Después de colocar el primer puerto bajo visión directa, se instaura el pneumoperitoneo a $12 \mathrm{~mm}$ de $\mathrm{Hg}$, colocando después los 5 puertos restantes, y conectándolos a los brazos robóticos. Puede usarse el gancho monopolar, la tijera fría o la pinza bipolar según las preferencias del cirujano. Se inicia la exploración transperitoneal con una óptica de $30^{\circ}$, comenzando la prostatectomía accediendo directamente al cuello vesical, sin obertura de la fascia endopélvica. La identificación del cuello vesical se facilita gracias a la tracción craneal de la vejiga por parte del asistente. Una vez seccionada la pared anterior del cuello hasta la sonda, el asistente tracciona de ésta para que el cirujano pueda seccionar la pared posterior del cuello. Con esta maniobra se accede a visualizar la fascia de Denonvilliers. La apertura de su hoja anterior permitirá entrar a disecar ambos conductos deferentes y vesículas seminales, cuya arteria puede ser clipada o coagulada. A continuación y gracias a la tracción superior de cada vesícula, se expondrá la hoja posterior del Denonvilliers, creando cómodamente un espacio con disección roma. Esta anatomización permitirá identificar y controlar óptimamente los pedículos laterales prostáticos, que podrán ser clipados o cauterizados con bipolar. Seguidamente, para la preservación de bandeletas neurovasculares, se incide en la fascia prostática anterior (velo de Afrodita), donde se busca un plano entre la fascia y la cápsula prostática. Este plano está más profundo que el complejo de Santorini, es prácticamente avascular, excepto en la parte anterior donde la fascia se fusiona con los ligamentos puboprostáticos y queda cubierto por el complejo dorsal. Los ligamentos se seccionan con 
tijera fría en su inserción en el ápex prostático, destacando que el complejo dorsal se puede controlar antes o después de la sección de la uretra, con un punto de transfixión dependiendo de si existe o no sangrado profuso. Para la anastomosis uretrovesical se utiliza una sutura continua de monofilamento 3/0 con 2 agujas, dejando una sonda Foley 20Fr.

Definimos el tiempo operatorio desde que el cirujano se coloca en la consola del robot hasta que se retira de la misma. Este tiempo no comprende la colocación del robot por un equipo de enfermería entrenado y la colocación trócares y brazos, que requiere de 20 a 40 minutos, destacando que el proceso se reduce de forma considerable a medida que el equipo auxiliar gana experiencia.

En cuanto a los resultados funcionales, definimos continente al paciente que no usa compresa o usa una de seguridad contra la pérdida ocasional de algunas gotas de orina, y consideramos potente al que es capaz de mantener una relación sexual, con la ayuda o no de inhibidores de la fosfodiesterasa 5 (PDE5).

Se analizaron el tiempo operatorio, las pérdidas hemáticas, la tasas de transfusión y conversión, las complicaciones intra y postoperatorias, la estancia hospitalaria y los días de sonda vesical. También se revisaron las tasas de márgenes quirúrgicos positivos (MQP) así como su localización. Con un seguimiento medio de 10.3 meses (6-16), también se evalúan la progresión de PSA y los resultados funcionales.

\section{RESULTADOS}

La edad media de los pacientes fue de 62 años (45-75). El peso medio de la glándula determinado por ecografía transrectal fue 38,2 g (15-55). La media de PSA total preoperatorio fue $6,56 \mathrm{ng} / \mathrm{mL}$ (4,31-16,44). Todos los pacientes tenían CaP clínicamente localizado ( $\leq \mathrm{T} 2 \mathrm{c}$ ). El estadio clínico fue $\mathrm{T} 1 \mathrm{c}$ en 88 pacientes, T1b en otro, obtenido después de una RTU de próstata, 7 pacientes T2a, 2 T2b y 2 T2c. La media de tiempo operatorio fue de 180 minutos (100-310), es decir, 3 horas (3h $31 \mathrm{~min}-5 \mathrm{~h}$ $10 \mathrm{~min})$. La pérdida hemática media fue de $210 \mathrm{~mL}$ (80-390), requiriendo en 2 casos transfusión sanguínea por lesión de los vasos epigástricos, que no requirieron reintervención. No hubo complicaciones intraoperatorias, reconversiones ni reintervenciones, destacando que en ningún caso se produjo lesión rectal. Como acontecimientos postoperatorios destaca un síndrome compartimental que provocó una necrosis músculo-cutánea, que tuvo que ser reparada mediante injerto; una retención aguda urinaria tras retirada de sonda vesical, que únicamente obligó a recolocarla durante una semana más; y 2 casos de parestesias de miembros superiores, por probable compresión de plexo braquial, que cedieron espontáneamente a los 2 meses de la intervención. La estancia hospitalaria media fue 3.7 días (221). A los primeros 40 pacientes se retiró la sonda vesical el día 14 después de la intervención, a los 60 restantes se retiró el día 8 , sin cistografía previa. Todos los pacientes reanudaron micciones espontáneas, excepto en el caso descrito previamente de la retención.

En relación a los resultados oncológicos, y después del análisis anatomo-patológico de las piezas quirúrgicas, los estadios patológicos fueron, pT2a en 24 pacientes, pT2b en 2, pT2c en 61, pT3a en 8, pT3b en 4 pacientes, y 1 pTO. Se detectaron 21 casos con MQP (21\%). La localización más frecuente fue postero-lateral. Se observaron $5 \mathrm{MQP}$ en pT2a, 1 en pT2b, 9 en pT2c, 3 en pT3a y 3 márgenes afectos en pT3b (Tabla 1). De los 100 pacientes, todos tenían un PSA indetectable en el primer control al mes de la intervención, pero 3 de ellos han presentado progresión bioquímica posterior (1 pT2c y 2 pT3b).

Tabla 1. Estadio patológico, márgenes quirúrgicos y progresión de PSA al año de los 100 primeros casos de PRRdaV

\begin{tabular}{lccc}
\hline $\begin{array}{l}\text { Estadio } \\
\text { patológico }\end{array}$ & $\begin{array}{c}\mathbf{N}^{\mathbf{0}} \\
\text { pacientes }\end{array}$ & $\begin{array}{c}\text { Márgenes } \\
\text { quirúrgicos }\end{array}$ & $\begin{array}{c}\text { Progresión } \\
\text { PSA al año }\end{array}$ \\
\hline pT0 & 1 & - & - \\
pT2a & 24 & 5 & - \\
pT2b & 2 & 1 & - \\
pT2c & 61 & 9 & 1 \\
pT3a & 8 & 3 & - \\
pT3b & 4 & 3 & 2 \\
TOTAL & 100 & 21 & 3 \\
\hline
\end{tabular}

Referente a la continencia, 69 de los 100 pacientes $(69 \%)$ fueron continentes antes de los 3 meses de la intervención, y el 91\% lo fueron a los 9 meses. El 9\% restante requieren el uso de compresas, destacando que 2 de ellos (2\%) presentan urgencia miccional, de los que uno de ellos padece enfermedad de Parkinson. En cuanto a la función sexual, y 
recordando que se realizó un intento de preservación de bandeletas (uni o bilateral) en todos los casos, de los 100 pacientes evaluados, 13 de ellos (13\%) presentaban disfunción eréctil preoperatoria. De los 87 restantes, 54 (62\%) conservaban potencia en el momento de la revisión, y 33 (38\%) pacientes presentaron disfunción eréctil postoperatoria.

\section{DISCUSIÓN}

La PRRdaV ha generado un gran interés en Urología, siendo la indicación quirúrgica más habitual en los servicios que disponen de robot. El impacto positivo de la robótica es cada vez más imparable, así en EE.UU, donde existe el mayor número de robots, en el 2005, el 20\% de las prostatectomías se realizaron con el robot Da Vinci, el 0,7\% con laparoscopia y el $79,3 \%$ por vía abierta.; en el 2006, supuso el 41.5\% con el robot, el 0,5\% con laparoscopia y el 58,5\% por vía abierta, y la datos recientes de 2007 muestran una superioridad para la robótica con el 63,0\%, siendo el 0,2\% para la laparoscopia y el $36,85 \%$ para la vía abierta (IntuitiveSurgical.com).

En este trabajo, revisamos nuestros primeros 100 procedimientos y comparamos nuestros resultados con series internacionales publicadas previamente.

En los trabajos con amplias revisiones de PRRdaV, el número de cirujanos que intervienen es variable, desde un único cirujano en la serie de Patel et $\mathrm{al}^{13}, 2$ cirujanos en otras dos series ${ }^{14,15}$ y 3 cirujanos en la amplia revisión de Menon et $\mathrm{al}^{9}$. En nuestra revisión intervinieron 5 cirujanos, 4 de ellos con experiencia laparoscópica, pero siendo para todos la curva de aprendizaje para PRRdaV. Esto explica los mayores tiempos operatorios en comparación con las series con menos cirujanos y mayor experiencia de cada uno. Nuestro grupo definió la curva de aprendizaje para PRRdaV en 20 casos, considerando que son los que un cirujano debe realizar para conseguir la suficiencia o maestría en una técnica quirúrgica con el mínimo de complicaciones ${ }^{11}$. Nuestra media de tiempo operatorio fue de 180 minutos, es decir, 3 horas. Para comparar datos, en nuestro caso debemos añadir de 20 a 40 minutos más de tiempo de colocación de trócares e instalación del robot, ya que el resto de series publicadas cuentan como tiempo operatorio desde la incisión cutánea inicial hasta el cierre de la piel. Patel et $\mathrm{al}^{13}$ informan de un tiempo operatorio de
141,2 minutos, El-Hakim et al ${ }^{16} 222$ minutos, Mikhail et al ${ }^{15}$ de 342 minutos para los 100 primeros casos y de 208 minutos en los siguientes, y en la amplia serie de Menon et $\mathrm{al}^{9}$ se pasa de 116 minutos en los primeros casos a 97 minutos en los 200 últimos casos de tiempo de prostatectomía, disminuyendo el tiempo de colocación de trócares y robot de 45 minutos en los primeros casos a 8 minutos en los últimos 200.

En relación a los resultados oncológicos, aunque la supervivencia libre de recurrencia de PSA (SLRP) es la medida óptima de control, en la mayoría de trabajos es todavía corto para establecer conclusiones. Por esta razón, y en espera de un mayor seguimiento, la tasa de MQP se está usando como parámetro de valoración oncológica. En nuestra serie, detectamos una tasa de MQP del 21\%, que estaría en el rango de las experiencias Europeas (22\%) ${ }^{17}$, mientras que Menon et al. reportan una tasa del 9\% inicial al $4 \%$ en los últimos $\operatorname{casos}^{9}$. Este mismo grupo, que tiene la mayor serie y seguimiento $(2.766$ casos a 5 años) informa de una SLRP del 84\% a 22 meses. En nuestra serie, de los 100 casos, todos tenían un PSA indetectable al mes de la intervención, pero 3 de ellos han presentado un progresión bioquímica posterior durante el seguimiento (SLRP 97\%). Del resto de series, sólo Patel et al. con 500 casos y un seguimiento medio de 9,7 meses, informa de un $95 \%$ de SLRP $^{13}$. Nuestra pérdida hemática media fue de $210 \mathrm{~mL}$, con 2 casos (2\%) de transfusión por lesión de vasos epigástricos. Estas pérdidas están en consonancia con el resto de series robóticas iniciales ${ }^{9,13-15}$. Ninguno de nuestros casos presentó complicaciones mayores, reconversiones ni reintervenciones. Destacan 2 casos de parestesias de miembros superiores, por probable compresión de plexo braquial, que cedieron espontáneamente. Mottrie et al. reportan el mismo trastorno en 3 pacientes, y desde entonces realizan medidas extras de seguridad para proteger los puntos de presión del paciente posicionado en Trendelenburg extremo ${ }^{14}$. Nuestra estancia hospitalaria media fue 3,7 días, mayor que la presentada en otras series, entre 24 horas en la serie de Menon et $\mathrm{al}^{9}$ a 1.5 días de media en el resto ${ }^{18,19}$. La mayoría de nuestros casos estuvieron 2 días ingresados, pero la estancia media es mayor al resto debido al ingreso de 21 días del paciente que presentó el síndrome compartimental. A nuestros primeros 40 pacientes se retiró la sonda vesical el día 14 después de la intervención, a los 60 
restantes se retiró el día 8 , sin cistografía previa. En el resto de series, se retira la sonda vesical a los 7 8 días, realizando cistografía en la mayoría de ca$\operatorname{sos}^{9,13-15}$.

La exquisita instrumentalización robótica que permite minimizar la disección del tejido periuretral y los ligamentos puboprostáticos explicaría que varios estudios apuntan a una mejor y precoz continencia mediante PRRdaV ${ }^{19-21}$. Los grupos de Menon, Mottrie y Patel informan de una continencia total en el 95-96\% de los pacientes a los 6 meses de seguimiento $^{12-14}$. En nuestro estudio el 91\% lo fueron a los 9 meses de seguimiento. En cuanto a la función sexual, los datos sobre erección postoperatoria a PRRdaV son todavía precoces. En algunas series, aproximadamente el 80\% de pacientes presentan erecciones (espontáneamente o con ayuda de inhibidores PDE5) con una media de seguimiento de 7.7 meses ${ }^{18,19}$. En nuestra revisión, de los pacientes potentes preoperatoriamente, el 62\% conservaban potencia en el momento de la revisión (10 meses), con un 38\% de disfunción eréctil postoperatoria en diferentes grados. Los grupos con mayor número de casos concluyen que la curva de aprendizaje para los resultados funcionales es más larga que para el resto de parámetros, e incluso debería ser valorada aparte cuando se realiza dicha curva en PRRdaV. También refieren que una meticulosa disección de las bandeletas neurovasculares sin el uso de cauterio, mejoraría estos resultados ${ }^{9}$. Este hecho explicaría los peores resultados funcionales de nuestra serie, debido a que ha sido la curva de aprendizaje robótica para 4 de los 5 cirujanos de la revisión, y al mayor uso de cauterio en nuestros primeros casos.

Nuestro grupo realizó todos los procedimientos mediante un abordaje transperitoneal. Algunos centros han iniciado recientemente la $\mathrm{PRRdaV}$ vía extraperitoneal. Estos autores concluyen que este abordaje es factible, con buena visualización y correcta maniobrabilidad del robot, evitando la cavidad peritoneal ${ }^{22}$. Además no parecen existir diferencias significativas en los resultados de ambas técni$\operatorname{cas}^{23}$.

Debido a la gran dependencia quirúrgica del robot, es importante valorar los posibles fallos mecánicos o de software que podrían evitar la continuación de la cirugía. Aunque en nuestra serie no hemos presentado ningún fallo del robot, Menon et $\mathrm{al}^{9}$ presentaron un $0,2 \%$ de fallos del robot $5 \mathrm{de}$ 2.766 casos). En 2 casos se pudo proseguir con la intervención, pero en el resto se finalizó la intervención por vía laparoscópica dada la imposibilidad de continuar vía robótica. Por esta razón, será importante informar preoperatoriamente a todos los pacientes de esta infrecuente posibilidad.

Por último, nos encontramos con el problema del coste en Europa, con sistemas de Salud Públicos diferentes a EE.UU. El coste de un robot es de 1.300.000 - 1.850.000 Euros, siendo los costes de mantenimiento aproximadamente el 10\% de esta suma. Además, el instrumental cuesta entre 1.000 y 1.500 Euros por caso. Aunque la PR laparoscópica podría llegar a asemejarse, en cuanto a costes, a la cirugía abierta, por el momento no será el mismo caso para la PRRdaV.

\section{CONCLUSIONES}

La PRRdaV es un procedimiento seguro, reproducible y que ofrece unos resultados oncológicos y funcionales muy prometedores con un abordaje minimamente invasivo. A pesar de incluir la curva de aprendizaje de 5 cirujanos, obtenemos una excelente tasa de continencia, y una buena función sexual. La mejora individual, con mayor experiencia, así como un mayor seguimiento, permitirá valorar la evolución de la técnica, así como de sus resultados.

\section{REFERENCIAS}

1. Bracarda S, de Cobelli O, Greco C, Prayer-Galetti T, Valdagni R Gatta G al. Cancer of the prostate. Crit Rev Oncol Hematol. 2005 Dec;56(3):379-396.

2. Parkin DM, Bray FI, Devesa SS. Cancer burden in the year 2000 The global picture. Eur J Cancer. 2001;Suppl 8:S4-66.

3. Han M, Partin AW, Pound CR, Epstein JI, Walsh PC. Long-term biochemical disease-free and cancer-specific survival following anatomic radical retropubic prostatectomy. The 15-year Johns Hopkins experience. Urol Clin North Am. 2001;28(3):555-565.

4. Badani KK, Kaul S, Menon M. Evolution of robotic radical prostatectomy: assessment after 2766 procedures. Cancer. 2007;110 (9): 1951-1958.

5. Patel VR, Shah K, Palmer KJ, Thaly R, Coughlin G. Roboticassisted laparoscopic radical prostatectomy: a report of the current state. Expert Rev Anticancer Ther. 2007;7(9):1269-1278.

6. Binder J, Kramer W. Robotically-assisted laparoscopic radical prostatectomy. BJU Int. 2001;87(4):408-410.

7. Menon M, Shrivastava A, Tewari A, Sarle R, Hemal A, Peabody $\mathrm{JO}$, et al. Laparoscopic and robot assisted radical prostatectomy: establishment of a structured program and preliminary analysis of outcomes. J Urol. 2002;168(3):945-949.

8. Tewari A, Peabody J, Sarle R, Balakrishnan G, Hemal A, Shri vastava A, et al. Technique of da Vinci robot-assisted anatomic radical prostatectomy. Urology. 2002;60(4):569-572.

9. Badani KK, Kaul S, Menon M. Evolution of robotic radical prostatectomy: assessment after 2766 procedures. Cancer. 2007;110 (9): 1951-1958. 
10. Villavicencio Mavrich H. Da Vinci advanced robotic laparoscopic surgery: origin and current clinical application in urology, and comparison with open and laparoscopic surgery. Actas Urol Esp. 2006;30(1):1-12.

11. Villavicencio Mavrich H, Esquena S, Palou Redorta J, Gómez Ruíz JJ. Robotic radical prostatectomy: overview of our learning curve. Actas Urol Esp. 2007 Jun;31(6):587-592.

12. Menon M, Shrivastava A, Kaul S, Badani KK, Fumo M, Bhandari M, et al. Vattikuti Institute Prostatectomy: Contemporary Technique and Analysis of Results. Eur Uro 2007;51(3); 648-658.

13. Patel VR, Thaly R, Shah K. Robotic radical prostatectomy: outcomes of 500 cases. BJU Int. 2007;99(5):1109-1112.

14. Mottrie A, Van Migem P, De Naeyer G, Schatteman P, Carpentier P, Fonteyne E.. Robot-assisted laparoscopic radical prostatectomy: oncologic and functional results of 184 cases. Eur Urol. 2007;52(3):746-750.

15. Mikhail AA, Orvieto MA, Billatos ES, Zorn KC, Gong EM, Brendler $\mathrm{CB}$, et al. Robotic-assisted laparoscopic prostatectomy: first 100 patients with one year of follow-up. Urology. 2006;68(6):1275-1279.

16. El-Hakim A, Leung RA, Tewari A. Robotic prostatectomy: a pooled analysis of published literature. Expert Rev Anticancer Ther. 2006;6(1):11-20.

17. Cathelineau X, Rozet F, Vallancien G. Robotic radical prostatectomy: the European experience. Urol Clin North Am. 2004;31(4):693-699. VIII.

18. Ahlering TE, Skarecky D, Lee D, Clayman RV. Successful transfer of open surgical skills to a laparoscopic environment using a robotic interface: initial experience with laparoscopic radical prostatectomy. J Urol. 2003;170(5):1738-1741.
19. Menon M, Tewari A, Baize B, Guillonneau B, Vallancien G. Prospective comparison of radical retropubic prostatectomy and robot-assisted anatomic prostatectomy: the Vattikuti Urology Institute experience. Urology. 2002;60(5):864-868.

20. Takenaka Takenaka A, Tewari AK, Leung RA, Bigelow K, ElTabey N, Murakami G, et al. Preservation of the puboprostatic collar and puboperineoplasty for early recovery of urinary continence after robotic prostatectomy: anatomic basis and preliminary outcomes. Eur Urol. 2007;51(2):433-440.

21. Ficarra V, Cavalleri S, Novara G, Aragona M, Artibani W. Evidence from robot-assisted laparoscopic radical prostatectomy: a systematic review. Eur Urol. 2007;51(1):45-55;

22. Joseph JV, Rosenbaum R, Madeb R, Erturk E, Patel HR. Robotic extraperitoneal radical prostatectomy: an alternative approach. J Urol. 2006;175(3 Pt 1):945-950.

23. Atug F, Castle EP, Woods M, Srivastav SK, Thomas R, Davis R. Transperitoneal versus extraperitoneal robotic-assisted radical prostatectomy: is one better than the other?. Urology. 2006;68 (5):1077-1081.

Correspondencia autor: Dr. Humberto Villavicencio Mavrich Servicio de Urología. Fundació Puigvert Cartagena, 340-350 - 08025 Barcelona Tel.: 934169700

E-mail autor: hvillavicencio@fundacio-puigvert.es Información artículo: Original - Cáncer de próstata Trabajo recibido: octubre 2008

Trabajo aceptado: noviembre 2008 